\title{
Absence of a causal relationship between environmental and body temperature in dairy cows (Bos taurus) under moderate climatic conditions
}

\author{
Wynand Verwoerd, Martin Wellby and Graham Barrell* \\ Agriculture and Life Sciences Division, P O Box 84, Lincoln University \\ Canterbury 8150, New Zealand
}

*Corresponding author: fax no.643 3253851, email barrell@lincoln.ac.nz

\begin{abstract}
1. Continuous body temperature records from dairy cows for 46 days of summer and contemporary data for climate temperature humidity index (THI) were analysed,
\end{abstract}

2. A large component of the body temperature data was not explained by changes in THI.

3. Outside a normal range of values (above 72 for THI and $39.05{ }^{\circ} \mathrm{C}$ for body temperature), there was evidence of a causal relationship with a time delay of about 120 minutes.

4. Both variables had a prominent circadian component, but these were more likely to be due to common and/or independent causes than to any direct relationship.

Keywords: Circadian rhythm; Core temperature; Dairy cows; Heat stress; Climate; Temperature rhythm

The definitive version is available from: www.sciencedirect.com/science/journal/03064565

\section{Introduction}

Continuous recordings of body temperature obtained from animals invariably show that the readings oscillate with time and these oscillations can be explained variously as being the result of changes in activity of the animal, changes in set point, changes in the thermal properties of the environment, changes in physiological processes within the animal or they may be entirely random events or combinations of any of these. In some cases the oscillations are randomly episodic; in others they may be regular, often with circadian rhythms. They may represent passive shifts in heat resulting from environmental or internal changes in thermal load, with time lags due to thermal inertia, or they may arise from shifts in active thermoregulatory adjustments. The latter can be explained by the current theory of thermoregulation which is based largely on the concept of a set point temperature that is maintained by the regulatory system. However, there is some uncertainty about the concept of a set point (Rifinetti ,1998; Briese, 1998) and this raises major questions about the validity of the current theory underlying thermoregulation. Also, this exposes researchers to difficulties in the interpretation of causes of change in body temperature of animals. Since 
there may be concerns about the welfare or productivity of farm animals in adverse climates, it is important that a clear picture of the relationship between thermoregulatory activity of such animals and thermal effects of the environment is developed. To examine this possible relationship, we tested the hypothesis that episodic changes in core body temperature can be explained by changes in the thermal environment by performing exploratory data analysis of continuously recorded body temperatures from lactating dairy cows and concomitant ambient climate measurements.

The analysis presented made no assumption about the existence or otherwise of a thermoregulatory set point. It simply compared, and looked for coordinated changes, in two sets of synchronous data: the body temperature $\left(\mathrm{T}_{\mathrm{c}}\right)$ values and values of the temperature humidity index (THI).

It was assumed that there is an underlying circadian rhythm in each of these data sets.

Whether the $T_{c}$ cycle is caused by the circadian cycle of the thermal environment, was left as an open question to be investigated from the data, although there are substantial counterindications to this proposition. Even if a statistical correlation between circadian components of $\mathrm{T}_{\mathrm{c}}$ and $\mathrm{THI}$ is found, that would not be convincing proof of a causal relationship because there are obvious common (and also independent) causes of the individual cycles. Therefore, to facilitate finding a correlation between non-circadian components, the first task of the analysis was to isolate and remove the circadian contribution from each of the time series.

A second confounding factor is the inevitable presence of random noise in both time series. While such random contributions will by definition not contribute to the statistical correlation between large data sets, visual comparison and recognition of episodic coordination are facilitated by eliminating or reducing noise, and was a second task of the data analysis. The remaining smoothed, non-circadian pair of temperature time series were then inspected for statistical correlation or other evidence of a $\mathrm{T}_{\mathrm{c}}$ response that is coordinated in time (possibly with a delay) with the stimulus supplied by a change of the THI.

\section{Materials and methods}

\subsection{Animals and experimental procedure}

Animals were mixed-age Holstein-Friesian x Jersey cows (Bos taurus) chosen at random from a herd of about 650 lactating dairy cows on the Lincoln University Dairy Farm in Canterbury, New Zealand. These cows experienced twice daily milking at approximately 0530 to $0830 \mathrm{~h}$ and 1430 to $1730 \mathrm{~h}$ New Zealand Daylight Time (NZDT = GMT $-11 \mathrm{~h}$ ) in a 
milking shed and walked to and from paddocks between milkings where they grazed on ryegrass/white clover pasture. At an afternoon milking, usually on Mondays, about 10 animals that were selected randomly from the herd received intravaginal temperature data loggers which remained in place for up to 7 days. These devices consisted of a temperature sensor and data logger ('Minilog', Vemco Limited, Nova Scotia, Canada) mounted on a progesterone-free $\mathrm{CIDR}^{\mathrm{TM}}$ (controlled internal drug release device, InterAg New Zealand Limited, Hamilton, New Zealand) and were inserted about $20 \mathrm{~cm}$ into the vagina. They were connected by a synthetic string to a plastic flap that was glued to the rump of the animal as a measure to retain the recorders if they came out of the vagina. This exercise was repeated weekly using mostly different animals from November 2004 to February 2005 (Southern Hemisphere summer). Climate data were recorded at a permanent weather recording station (Broadfields station, National Institute of Water and Atmosphere Research Limited, New Zealand) situated about $3 \mathrm{~km}$ from the study site. Data from this recording station had been calibrated previously as being indistinguishable with data from a portable station set up at the study site.

The animal procedures used were all approved by the Lincoln University Animal Ethics Committee.

\subsection{Data analysis}

After downloading of data from data loggers onto a computer, intravaginal temperatures of the cows, hereafter called body temperature, were averaged for all cows measured at any one time (i.e. 8 to 10 cows) to give a single value, $\mathrm{T}_{\mathfrak{c}}$, that is considered to be representative of their core body temperature. This was repeated at fixed intervals of 10 minutes, giving 144 measurements per day and a total of about 46 days were sampled more or less randomly from a total summer period of 110 days (from 15 November 2004 to 14 February 2005). Values for different days were joined together sequentially to give a continuous progression of the clock time. From the climate station recordings, concomitant data were collected for ambient temperature $\left(\mathrm{T}_{\mathrm{a}}\right.$, degrees Celsius $)$ and dew point temperature $\left(\mathrm{T}_{\mathrm{dp}}\right.$, degrees Celsius $)$ and converted to a temperature humidity index (THI) using the following equation: $\mathrm{THI}=\mathrm{T}_{\mathrm{a}}+$ $\left(0.36 \times \mathrm{T}_{\mathrm{dp}}\right)+41.2$ (Mayer et al., 1999).

Two main tools were used for analysing the data. First, for each $\mathrm{T}_{\mathrm{c}}$ and THI value, the deviation from the average over the complete data set, and scaled by the corresponding standard deviation, was calculated. From this a scatter plot in which each point represents a synchronous $\left(\mathrm{T}_{\mathrm{c}}, \mathrm{THI}\right)$ pair of scaled deviation values, was made. Visual interpretation of the 
deviation scatter plot was supplemented by also performing spectral (Fourier) analyses of each of the two time series.

The data analysis was performed using a Microsoft Excel spreadsheet, and spectral analysis in this software package is limited to a maximum of 4096 data values. To accommodate that, the total data set was at this point divided into two overlapping sets of 4096 values for each of $\mathrm{T}_{\mathrm{c}}$ and THI. The earlier set was used to set up the separation into circadian, noise, and smoothed non-circadian components, and applying that to the later data set gives an indication of whether such a separation has predictive value.

To achieve the separation of the components, the first step was to average all values measured over the whole period at the same clock time each day. Inspection of the spectra for the two circadian averages showed a high contrast between the amplitudes of the prominent frequency peaks and those of the background. This allowed the small residual noise in the circadian components to be removed by filtering out all frequencies for which the amplitude was less than about $1 \%$ of the dominating peak. This provided the smoothed circadian variation shown in Figure 2 in the next section.

To investigate a possible causal relationship, a scatter plot of the circadian variation was made and the correlation coefficient between the circadian $\mathrm{T}_{\mathrm{c}}$ and THI components calculated. The behaviour of the correlation coefficient whilst varying the time delay of $T_{c}$ relative to THI, was monitored to determine if there was a phase relationship between them. The next step in the analysis was to subtract the smoothed circadian contribution from each of the raw datasets. The remainder was again subjected to spectral analysis. As the contrast between peak signals and background was not as clear-cut as with the circadian component, trials were made with different filtering thresholds, guided by checking that subtracted noise showed an approximately normal distribution, and also that correlation between $\mathrm{T}_{\mathrm{c}}$ and THI noise remained negligible. This led to the selection of values that produced a relatively smooth time variation for the each of the non-circadian components while retaining all features that seemed significant in the unfiltered data. The smoothed values were then combined into a scatter plot as before and analysed for coherent scatter plot behaviour, and for correlation and phase shift effects as described above for the circadian component.

\section{Results and discussion}

A first view of the relationship between the raw $\mathrm{T}_{\mathrm{c}}$ and THI values (before separation into components) is shown by the scatter plot in Figure 1. Note that while time information is not explicitly shown in the plot, the fact that the plotting routine connects plotted points by lines 
according to their data sequence, gives implicit information about the time sequence of individual measured pairs.

Various types of relationships between variables can be visually identified from a plot of this type. Perfectly correlated cyclical values with the same frequency of oscillation will give a diagonal straight line, while a phase shift between them changes this to an ellipse or circle, or to a more complicated Lissajous figure in case different frequencies are involved. In contrast, uncoordinated random values give a symmetrical spread around the origin, with the density decreasing away from the origin. The more realistic situation of random noise superimposed on an underlying cyclic behaviour, would give a cloud of points distributed about e.g. an elliptical shape.

The figure shows that for most points in our data there is no discernible coherent relationship, as the points appear randomly distributed about the means and fall within the 2 to 3 standard deviations circle that would be expected of normally distributed random data. However, there are at least two episodes where a short sequence of points project well out of the random cloud, and the fact that these both form rough loops gives an early indication that there might be a coherent phase relationship between the environmental stimulus (THI) and the physiological response $\left(T_{c}\right)$ during these episodes. The small calculated correlation coefficient of 0.13 between the two sets of data confirms that there is no evidence of a significant overall coordination of body temperature with the heat index, but is consistent with such coordination in a small subset of the data.

The smoothed circadian component of each of the two variables is shown in Figure 2. The curves shown in fact differ only marginally from those without applying smoothing. Also, there is very little change if the averaging is done over a small subset of the days in the dataset. For the $\mathrm{T}_{\mathrm{c}}$ profile, that is a reflection of the exceptional robustness (Refinetti, 2003) of the circadian rhythms observed in cattle (Piccione et al., 2003), which was found to be more robust than that of any mammalian species previously studied.

The shape of the THI curve is very similar to the daily outdoor temperature profile reported in a study of circadian rhythms in cows by Bitman et al. (1983). The maximum value (acrophase) of $\mathrm{T}_{\mathrm{c}}$ is a flat peak of $38.8^{\circ} \mathrm{C}$ stretching from 1730 to $1830 \mathrm{~h}$ NZDT and lags behind that of the THI curve at $1530 \mathrm{~h}$. The reverse holds for the daily minimum: the minimum $\mathrm{T}_{\mathrm{c}}$ value of $38.24{ }^{\circ} \mathrm{C}$ is reached at $0500 \mathrm{~h}$, which is earlier than the THI minimum reached at $0640 \mathrm{~h}$. To quantify the time differences as an overall phase difference, the correlation was calculated while shifting the $T_{c}$ values forward in time in steps of 10 minutes (the resolution of the data set). The correlation coefficient was found to be maximised to a 
value of 0.86 by a shift of 160 minutes, but was in fact quite insensitive to the actual time lag, giving values above 0.82 for all time shifts between 100 and 220 minutes.

Both this insensitivity and the differences in detail between the circadian $T_{c}$ and THI profiles argue against a close causal relationship, despite the quite high statistical correlation between them. Even when time shifted to maximise the correlation, there are periods when rapid changes in $T_{c}$ coincide with relatively stable THI values, and vice versa. These observations are consistent with assuming that the circadian body temperature cycle is independent of the thermal environment, as foreshadowed in the introduction.

The quite marked oscillation of $\mathrm{T}_{\mathrm{c}}$ in the early morning hours remains to be explained, but as this phenomenon was not observed in a separate set of measurements we performed on nonlactating cows, this may be caused by metabolic effects of milk production and/or the daily milking schedule to which these lactating cows were subjected.

Neither of the circadian profiles reflects a cosine shape and they would not be adequately modelled by the commonly used cosinor model (Nelson et al., 1979). Using the full spectral representation instead, ensures that a periodic repetition of the exact circadian profiles, shown in Figure 2, are subtracted in order to isolate the non-circadian contribution to each time series. Scatter plots of the smoothed non-circadian components of $\mathrm{T}_{\mathrm{c}}$ and THI are shown in Figure 3.

The first plot shows the values as calculated, in which each point represents a synchronous pair of values. As for the raw data in Figure 1, most of the data appear as an uncoordinated mass within two standard deviations of the centre.

However, there are two prominent loops projecting far out of this, as well as a few smaller ones of which the most noticeable reaches slightly above 3 standard deviations on the $\mathrm{T}_{\mathrm{c}}$ axis. It is worth noting that the time order in which the peak THI and body temperature peak occur, is significant if a raised THI is to be interpreted as the cause of a high $\mathrm{T}_{\mathrm{c}}$ value. The most plausible scenario, is where the $T_{c}$ peak follows that of the THI, after a time delay caused by the thermal inertia associated with the limited heat transfer rate and large body mass of a cow. That is the situation represented by the two largest loops, as indicated on Figure 3 by arrows representing the actual time sequence of the data. By contrast, the arrow for the $3^{\text {rd }}$, smaller loop shows that it is traversed in the opposite direction so that the body temperature peaks first in that case. To explain that as a causal relationship, one would have to invoke an active cooling response that is sufficiently drastic not only to mitigate the rise in body temperature, but to reverse it and force $T_{c}$ down at a time when the external THI is still rising fast. Not only does there not seem to be evidence for such a physiological response in 
general, but inspection of the figure shows that there is no indication of a credible trigger for it as the THI values throughout the duration of the $3^{\text {rd }}$ loop remain well within the normal range of variation. It is concluded that this $3^{\text {rd }}$ loop is more plausibly ascribed to a coincidence of independent trends in the two variables. Similar observations hold for the other minor loops visible in the figure, reinforced for those projecting below by noting that for them it is a minimum of $\mathrm{T}_{\mathrm{c}}$ that coincides with high THI values, and this is even less likely to be cause and effect.

To investigate if there is a coherent phase relation between the values, the time shift that maximises the correlation coefficient between the complete sets of $\mathrm{T}_{c}$ and $\mathrm{THI}$ values was calculated, and found to be 130 minutes. As shown in the second plot of Figure 3, that reduces the two prominent loops nearly to straight lines. By contrast, the smaller loops are widened, as also happens for any other time delay that is introduced. That is another indication that the small loops are 'time reversed' in terms of a causal connection and should be discounted for that purpose.

If the two large loops also only represented random coincidences of trends that were by chance in the right time order they would be expected to require different phase shifts to align them. But as they are both in fact aligned by the same shift of 130 minutes, it is a strong indication that they also share a common cause. Moreover, the value is also consistent with the delayed response time range found for the circadian cycle. Taken together with the exceptionally large amplitudes of the temperature deviations during the two episodes associated with the large loops, the most reasonable explanation is that there is a trigger level of the THI, beyond which the normal temperature regulation mechanisms of the cow is unable to cope and this caused the body temperature to rise by almost 3 times the normal range of variation in the more severe of the two episodes.

In order to find a practical guideline for identifying environmental conditions that lead to thermal stress, it is of interest to determine a value for the THI trigger level, and also a corresponding threshold for the body temperature that indicates such stress. Inspection of Figure 3 suggests that 2.5 standard deviations on either axis is a reasonable estimate of both threshold values. While the figure only shows the non-circadian component, similarly applying 2.5 standard variations to the full data set yields the values $\mathrm{THI}=72$ and $\mathrm{T}_{\mathrm{c}}=39.05$ as the respective thresholds.

To test this explanation, the pair of smoothed time series were each subdivided into the subset of 'ordinary' values below the threshold and the subset of 'exceptional' values that are above it. For the quoted threshold values, there were $101 \mathrm{THI}$ values and $71 \mathrm{~T}_{\mathrm{c}}$ values in the 
exceptional set, out of a total of 4096. The exceptional values were highly correlated, with a coefficient value of 0.91 attained for a delay of 120 minutes. Such a high correlation for isolated events not related to the daily cycle is hard to explain in any other way than as a direct cause and effect. If it is, one would expect the correlation to depend quite closely on the actual time delay. That is indeed what is found; changes in the special values correlation are about 5 times larger than corresponding changes of the circadian correlation, for similar percentages of change of the delay values in both cases. The value of 120 minutes seems the best measurement of the delayed response possible from the data.

In contrast, the remainder i.e. 'ordinary' points are weakly anti-correlated with a coefficient (0.2 ). The time delay hardly affects this value; similar values are obtained for delays between 0 and 500 minutes. This strongly suggests that for THI values below the trigger level, the body temperature is regulated independently of the THI. Visual inspection of the time series confirms that even after compensating for the delay, there are times when the body temperature trends up while THI trends down or vice versa, as a negative correlation indicates.

The focus of this work is on interpreting the relationship between body temperature $\left(\mathrm{T}_{\mathrm{c}}\right)$ and temperature humidity index (THI) values for lactating cows, observed under normal farm production conditions. While several other studies have been performed of circadian temperature cycles and the effects of heat stress on the physiology and production parameters of dairy cows, comparisons between these are not as simple as for laboratory experiments on small animals where external conditions can be standardized. In particular, the most interesting results obtained here relate to a couple of isolated episodes where exceptional behaviour was found, and so are not readily comparable.

Nevertheless an overall assessment of how the results reported here relate to previous experimental work, can be made by comparing some general parameter values.

An early study by Maust et al. (1972) yielded a mean rectal temperature of $39.4{ }^{\circ} \mathrm{C}$ at average daily THI values of $75 \pm 4.2$ during a 52 day experimental period, and shows a substantial correlation coefficient of 0.67 between the daily THI and $\mathrm{T}_{\mathrm{c}}$ values. The mean temperature value is considerably higher than the average of $38.48{ }^{\circ} \mathrm{C}$ obtained from our complete data set, but so is the THI when compared with our mean THI value of 60.5. In fact, when we restrict the calculation of mean values to the two episodes where we found good correlation, these are $39.5^{\circ} \mathrm{C}$ at a mean THI of 76 and $39.3{ }^{\circ} \mathrm{C}$ at a mean THI of 74 respectively. These results agree well with those of Maust et al. (1972), although it should be noted that they used 
a slightly different definition of the THI, basing it on wet and dry bulb temperatures rather than the dew point definition that we use.

A subsequent study also using the Maust definition of THI, was done by Araki et al. (1984). They measured vaginal temperature over a period of 17 summer days and found average values of $39.0{ }^{\circ} \mathrm{C}$ and $38.7{ }^{\circ} \mathrm{C}$ respectively for lactating and non-lactating cows, at an average daily THI of 75.3. These values are somewhat lower than the ones discussed above, while the temperature differential of $0.3{ }^{\circ} \mathrm{C}$ between lactating and non-lactating cows is in the same direction but larger in size than the $0.15^{\circ} \mathrm{C}$ differential we found by comparing the values discussed here with additional measurements we made on non-lactating cows.

The concept of a threshold THI value to determine effects on dairy cow productivity, has become widely accepted since it was first introduced by Johnson et al. several decades ago. It was suggested (Johnson et al., 1962) that milk yield declines slightly when the THI exceeds 72 and sharply when 76 is exceeded. Subsequent work (see West, 1999 for a review) established differences in the details of productivity responses according to breed, stage of lactation, and nutrition; and others, e.g. Ravagnolo (1999) found improved correlations with either minimum or maximum THI rather than average values. Nevertheless, critical values around 72 seem to have been generally accepted as determining the onset of heat stress in lactating cows. In the study of Mayer et al. (1999) for example, a value of 72 is proposed as appropriate for unshaded conditions, while the presence of shade can raise the threshold for productivity losses to a value of 76 . The value of 72 obtained in our study agrees well with those quoted above, but it should be emphasised that it was obtained independently, as the value that separates the correlated body temperature response we observed in isolated high THI episodes, from the uncorrelated values under moderate heat conditions.

Some caution is in order in the comparison of these THI thresholds, as several different formulas have been used to calculate the THI in the literature on heat stress in cattle. For example, in addition to the two different formulas discussed already, another version based on the percentage humidity was used by Ravagnolo et al. (2000). While these formulas are intended to be equivalent, the relationships between the various quantities they contain are not simple and the equivalence can only be assumed over limited parameter ranges. As detailed in a review by Lee (1980) the calculation of a heat index has a long history and further development is continuing, so that heat index values currently in use by meteorology services are often far more complex than the formula used in this and other animal heat stress studies. Modern heat index calculations (e.g., Matzarakis, 1999) are explicitly based on human thermal physiology and interaction with the environment, and even in the simpler 
formulas such as the one we used the coefficient values reflect this perspective. They may not be optimal for representing the heat response of large animals.

Another point of difference between our work and the productivity studies quoted above, is that we collected synchronous data at short time intervals for both THI and $\mathrm{T}_{c}$, while the previous work used daily averages and/or maxima and minima only. This is clearly not enough for taking either the circadian component or time lag effects into account as was done in this study. The need for frequent observations in circadian rhythm studies was stressed by Lefcourt et al. (1999) and also an earlier study by Bitman et al. (1984) recorded body temperature at closely spaced time intervals over periods of 2 days and 5 days respectively. Both of those studies were conducted on cows held in environment chambers with controlled temperature and artificial illumination cycles, so the results are not strictly comparable with those reported in this article. In the earlier study, an average difference of $1.25{ }^{\circ} \mathrm{C}$ between daily minimum and maximum was reported, while the later study gives an amplitude of 0.34 ${ }^{\circ} \mathrm{C}$ from a cosinor fit. The difference is presumably largely accounted for by the smoothing inherent in the cosinor model. Compared with these, the $0.55{ }^{\circ} \mathrm{C}$ range of variation shown e.g. in Figure 2 is in reasonable agreement, taking into account that our value is also smoothed but follows the actual temperature values much more closely than a cosinor model. Another intriguing observation is that in both of the quoted studies an ultradian oscillation was reported, with a period in the range 80-120 minutes (Bitman et al., 1984) or 100-175 minutes (Lefcourt et al., 1999). In our spectral analysis of the circadian component there are several peaks in these time ranges that stand out from the background noise, although they are small compared with the prominent peaks centred at 24, 12 and 8 hour periods. However, the combined effects of the finite spectral width of the dominating peaks and these smaller ones is to create a short period (roughly 120 minutes) oscillatory behaviour that is most clearly visible in the early morning, low temperature phase of the circadian body temperature profile shown in Figure 2.

Another recent study of circadian rhythms in cattle (Piccione et al. 2003) measured temperature values at 3-hourly intervals in both newborn calves and adult non-lactating cows for a period of 60 days. The climate in this study, in Sicily, is more directly comparable to ours in New Zealand than that in much of the published heat stress studies which were done in tropical climates. Piccione et al. (2003) recorded a mean level of $38.3{ }^{\circ} \mathrm{C}$ for the mature rhythm, and a $1.4{ }^{\circ} \mathrm{C}$ range of variation. This mean value is $0.2{ }^{\circ} \mathrm{C}$ lower than ours, in keeping with the difference we measured between lactating and non-lactating cows. The range of variation also is close to the overall variation of $1.35^{\circ} \mathrm{C}$ in our data, but that includes the two 
extreme episodes we observed as well as random noise; the smoothed circadian variation that we extracted only varies by $0.55^{\circ} \mathrm{C}$ as shown in Figure 2 . Due to the comparatively low time resolution in the Piccione et al. study, a detailed comparison of the circadian temperature profile is not possible, but they report an acrophase at 18:40 \pm 1 hour, in good agreement with the $17: 30$ to $18: 30$ range recorded here..

\section{Conclusions}

The data used in our study combine the advantages of a long duration (110 days) typical of heat stress studies and a high time resolution (10 minutes) typical of circadian rhythm studies. While it may seem more natural to study heat stress in a tropical environment, as done by most previous authors, the milder New Zealand climate creates the unique feature that our dataset contains isolated heat stress episodes superimposed on a background of normal variation. While the unpredictability of the episodes complicates data gathering, it makes it possible to identify time delays between climate and body temperature changes and at a more basic level, the presence or absence of a cause and effect relationship between these phenomena.

In order to exploit these features of our data, a new method of analysis was introduced in the form of deviation scatter graphs, and combined with more conventional spectral analysis, leading to a clear distinction between the circadian component of variation in both time series and the non-circadian part that includes the episodes where excursions outside of normal ranges were found.

Another important outcome is the finding that temperature regulation in cows in effect isolates the body temperature from the thermal environment under moderate conditions, but when a threshold THI value of 72 is exceeded this mechanism does not cope any more and the body temperature rises in response to the excess but with a time delay of 120 minutes. While this idea is to some extent implicit in the work on milk production under heat stress previously published, the ability to separate circadian and episodic changes and study their respective time correlations allowed us to reach a more explicit conclusion about it from our results.

A logical next step in extending this work and further confirm our interpretation, is to investigate if the magnitudes of the rise in $\mathrm{T}_{\mathrm{c}}$ and $\mathrm{THI}$ above their respective thresholds can be quantitatively related in a consistent way. For the two episodes that we observed, it turns out that indeed the larger body heat response occurred when the THI impulse was the greatest, as would be expected. 


\section{Acknowledgements}

This study was funded in part by Dairy InSight of New Zealand via a subcontract between ourselves and AgResearch, New Zealand. We thank Florian Bouvier and Amy Smaill for their assistance and the staff and management of the Lincoln University Dairy Farm for providing animals and facilities.

\section{References}

Araki C.T., Nakamura R.M., Kam L.W.G., Clarke N., 1984. Effect of lactation on diurnal temperature of dairy cattle in hot environments. J. Dairy Sci. 67, 1752-1760.

Bitman, J., Lefcourt, A., Wood, D.L., Stroud, B., 1983. Circadian and ultradian temperature rhythms of lactating dairy cows. J. Dairy Sci. 67, 1014-1023.

Briese, E., 1985. Rats prefer ambient temperatures out of phase with their body temperature circadian rhythm. Brain Res. 34, 389-393.

Briese, E., 1998 Normal body temperature of rats: the setpoint controversy. Neurosci.

Biobehav. Rev. 22, 427-426.

Johnson, H.D., Ragsdale, A.C., Berry, I.L., Shanklin, M.D., 1962. Temperature-humidity effects on milk production of Holstein cattle. Univ. Missouri Coll. Agric. Res. Bull. 791, 1-39 Lee, D.H.1980 Seventy five years of searching for a heat index. Environ. Res. 22, 331-356. Lefcourt, A.M., Huntingdon, J.B., Akers, R.M., Wood, D.L., Bitman, J.,1999. Circadian and ultradian rhythms of body temperature and peripheral concentrations of insulin and nitrogen in lactating dairy cows. Dom. Anim. Endocr. 16, 41-55.

Mayer, D.G., Davidson, T.M., McGowan, M.R., Young, B.A., Matschoss, A.L., Hall, A.B., Goodwin, P.J., Johnson, N.N., Gaughan, B., 1999. Extent and economic effect of heat loads on dairy cattle production in Australia. Aust. Vet. J. 77, 804-808.

Maust L.E., McDowell R.E., Hooven N.W., 1972. Effect of summer weather on performance of Holstein cows in three steps of lactation. J. Dairy Sci. 55,1133-1139.

Matzarakis A., Mayer H., Iziomon M.G., 1999, Applications of a universal thermal index: physiological equivalent temperature. Int. J. Biometeorol. 43,76-84.

Nelson, W., Tong, Y.L., Halberg, F., 1979. Methods for cosinor-rythmometry. Chronobiologia 6, 305-323.

Piccione, G., Caola, G., Refinetti, R., 2003. Daily and estrous rhythmicity of body temperature in domestic cattle. Bio Med Central Physiol. 3, 7. 
Ravagnolo O., Misztal I., Hoogenboom G., 2000. Genetic component of heat stress in dairy cattle, development of heat index function. J. Dairy Sci. 83, 2120-2125.

Refinetti, R.D., Menaker, M., 1992. The circadian rhythm of body temperature. Physiol.

Behav. 51, 613-637.

Refinetti, R., 1996. Rhythms of body temperature and temperature selection are out of phase in a diurnal rodent, Octodon degus. Physiol. Behav. 60, 959-961.

Refinetti, R.,1996. Comparison of the body temperature rhythms of diurnal and nocturnal rodents. J. Exp. Zool. 275, 67-70.

Refinetti, R., 1998. Homeostatic and circadian control of body temperature in the fat-tailed gerbil. Comp. Biochem. Physiol. 119A, 295-300.

Refinetti, R., 2003. Non-stationary time series and the robustness of circadian rhythms. J. Theor. Biol. 227, 571-581.

West J.E., 1999. Nutritional strategies for managing the heat-stressed dairy cow. J. Anim. Sci. 82, 21-35. 


\section{Normalised deviation scatter}

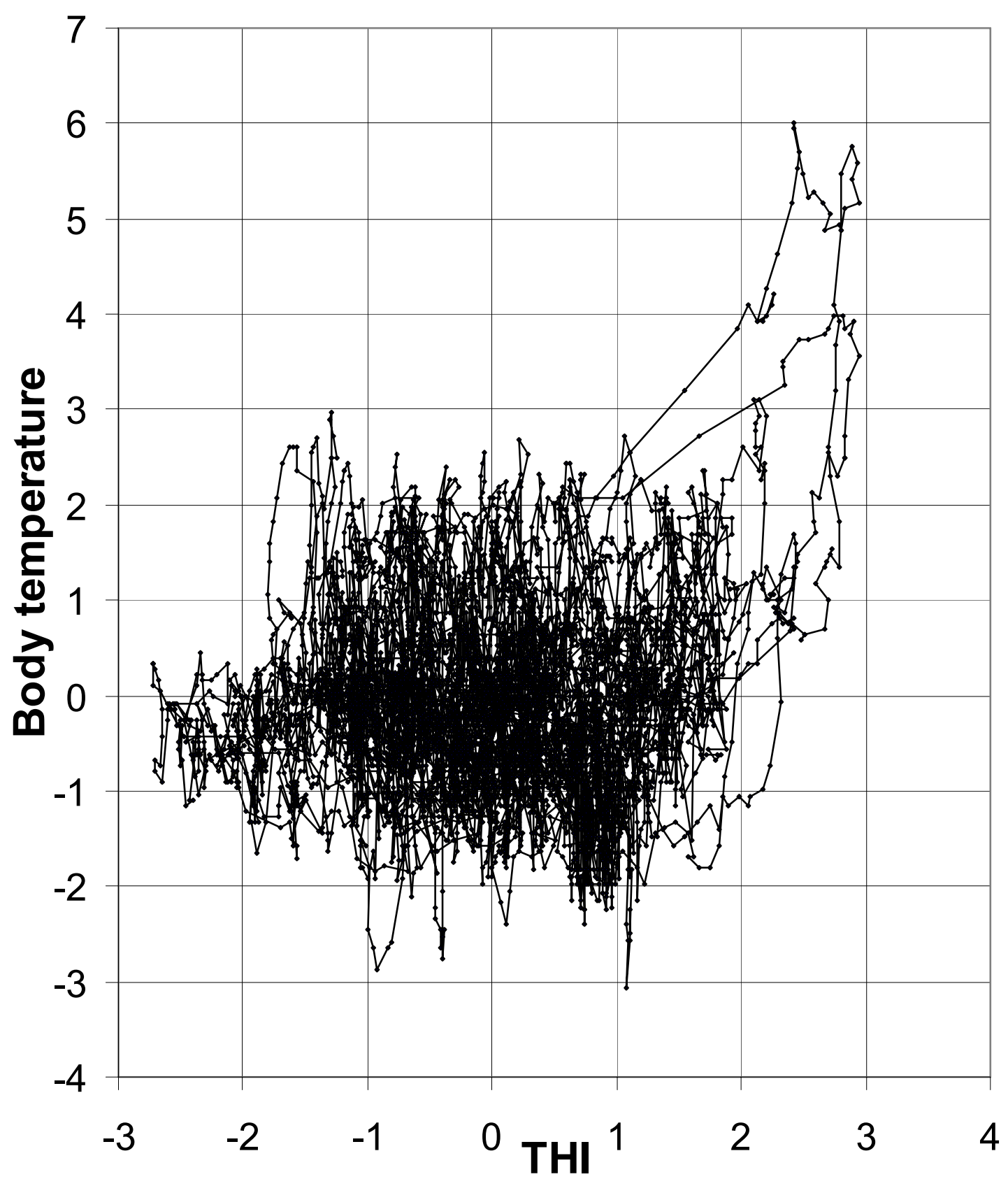


Smoothed daily average temperature

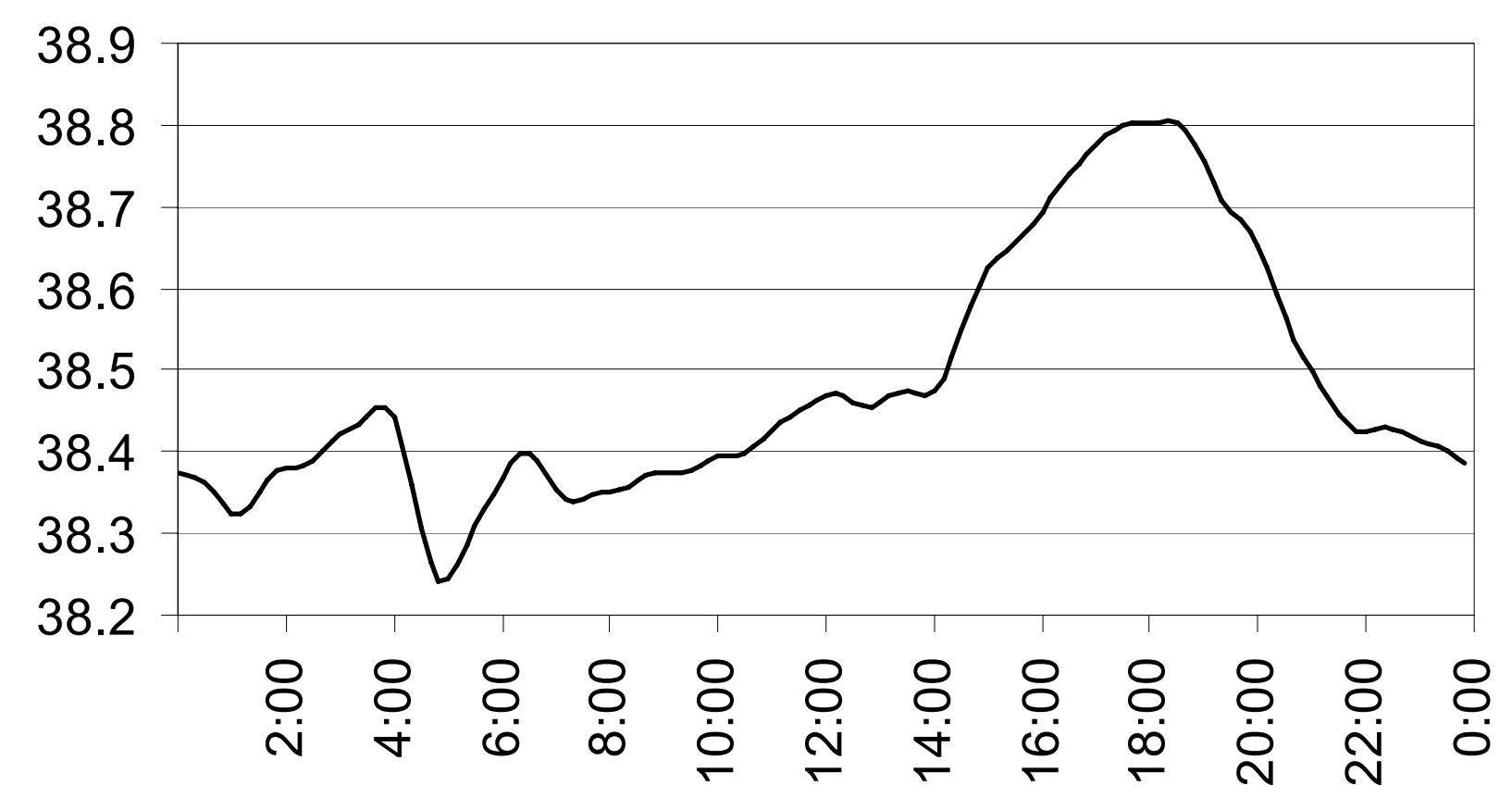

Smoothed daily average THI

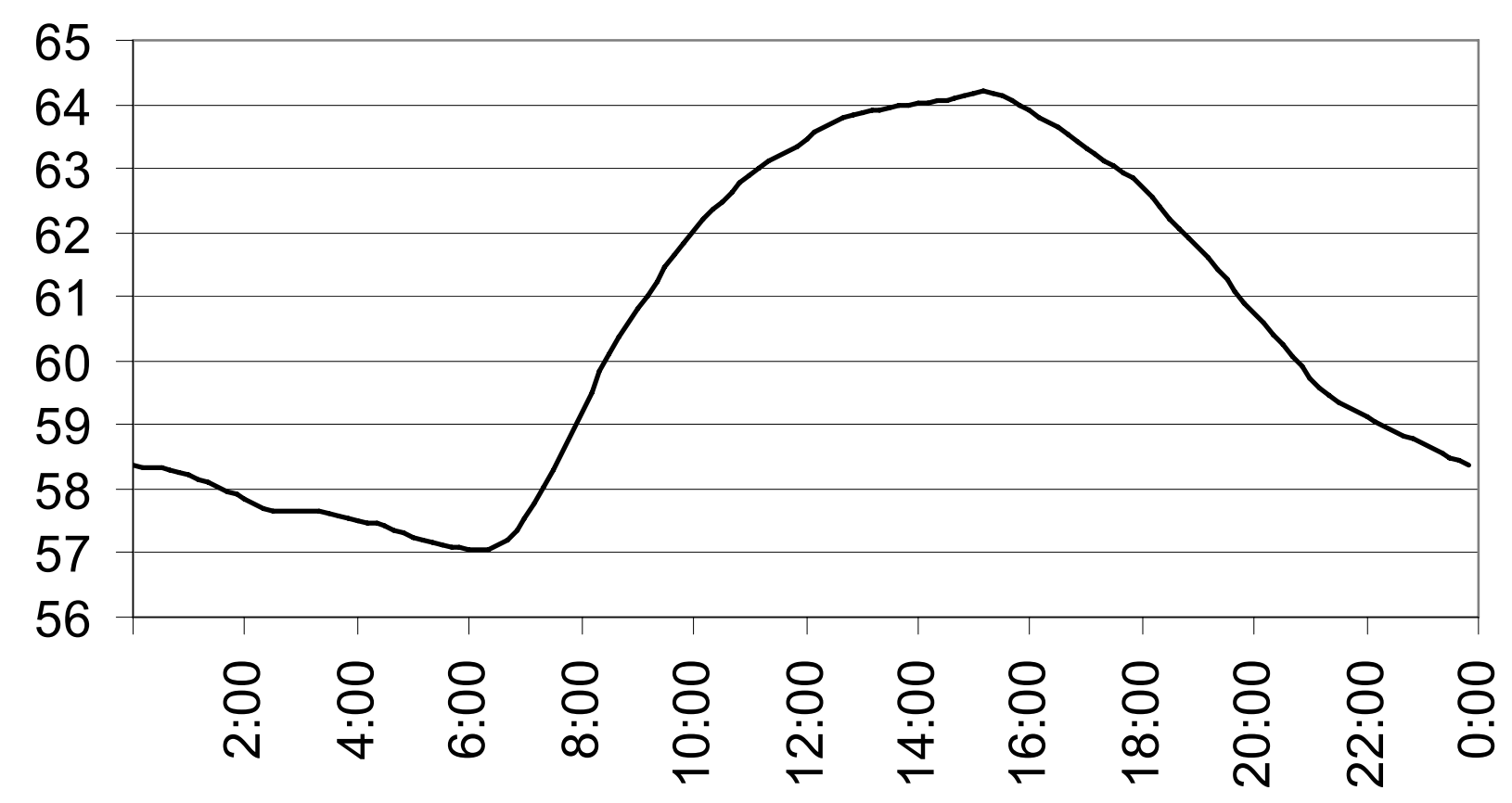




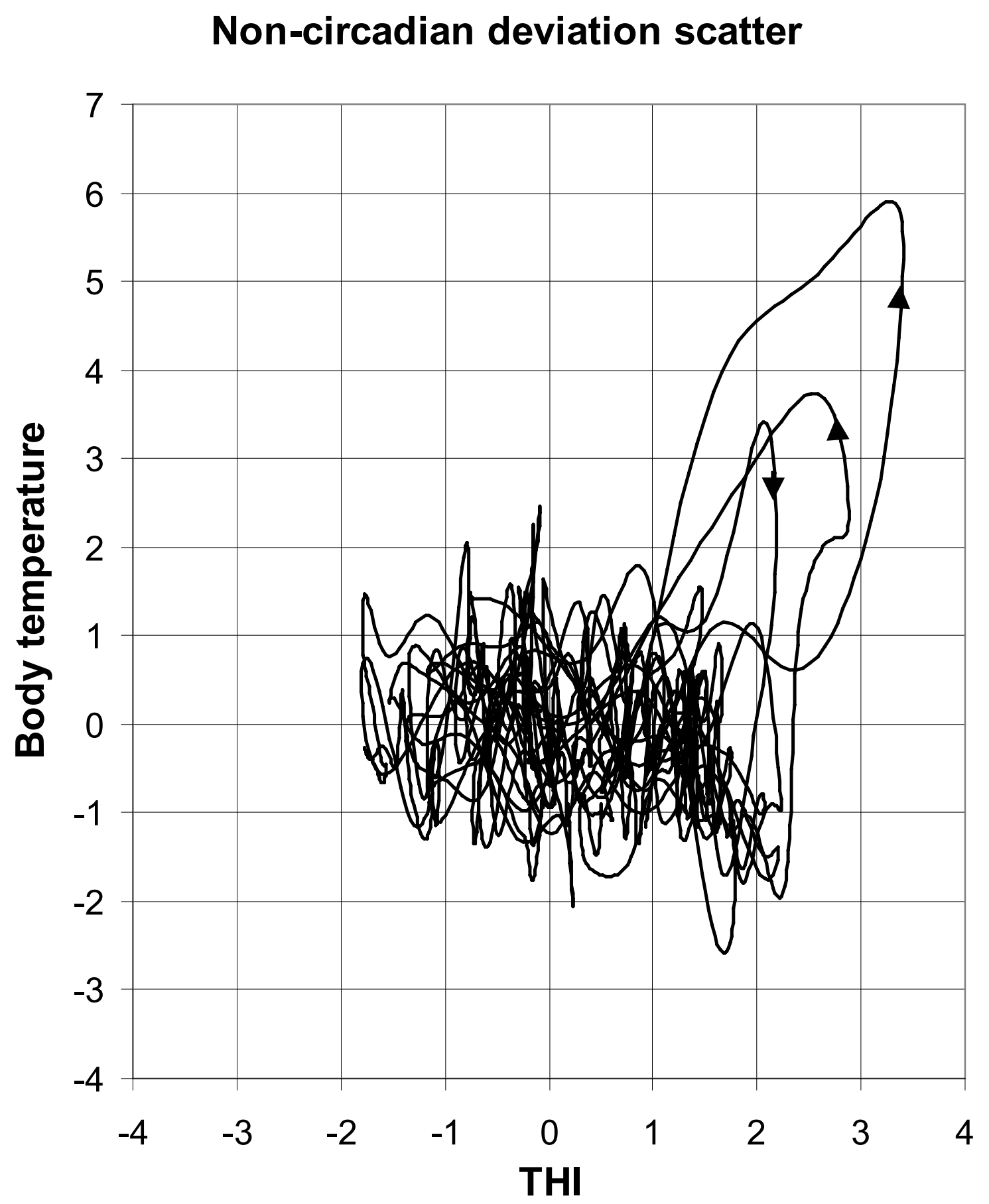


Time delayed non-circadian scatter

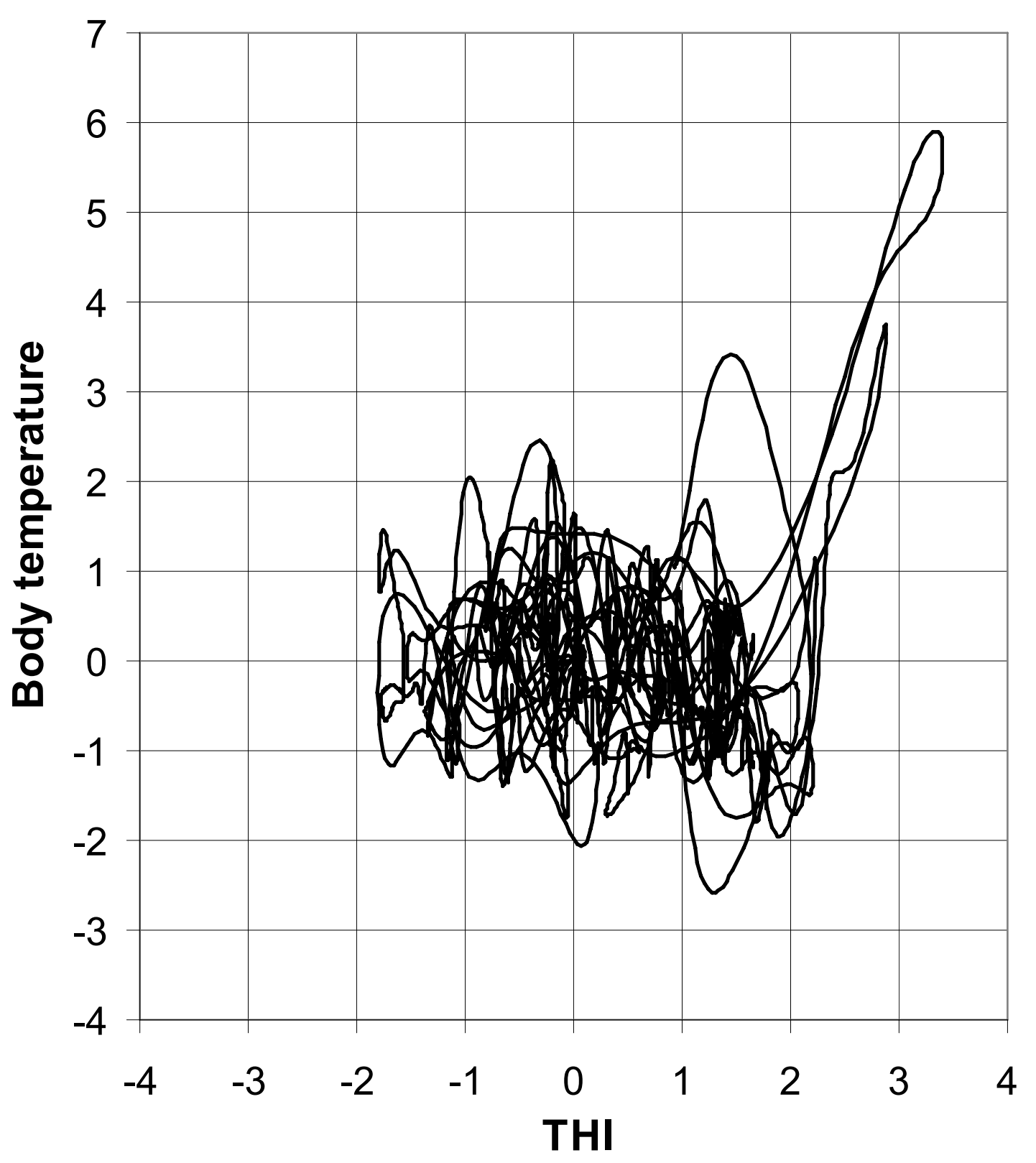




\section{Figure Captions}

Fig. 1: Scatter plot of body temperature and THI deviations from average, normalised to their standard deviations.

Fig. 2: Time profile (24-hour clock time) of the circadian variation of body temperature (in ${ }^{\circ} \mathrm{C}$ ) and heat index THI, as obtained by averaging all data measured at the same clock time each day, and after removal of residual noise by spectral filtering.

Fig. 3: Scatter plots of normalised deviations of the smoothed non-circadian components of body temperature and THI; left, synchronous data, and right, after introduction of a 130 minute time delay. 